\title{
Motion-Dependent Effects of Functional Magnetic Resonance Imaging Preprocessing Methodology on Global Functional Connectivity
}

\author{
Matthew N. DeSalvo
}

\begin{abstract}
Background: While functional magnetic resonance imaging (fMRI) has become an established noninvasive tool for studying brain activity in both healthy and diseased states, no broad consensus has been reached regarding preprocessing methodology. Furthermore, the relationship between variations in preprocessing and functional connectivity (FC) networks remains incompletely understood.

Purpose: The aim of this study was to relate FC to (1) choices in preprocessing methodology and (2) subject motion.

Methods: Clinical and MRI data were analyzed from healthy subjects acquired as part of the Autism Brain Imaging Data Exchange (ABIDE). Data were obtained from 508 healthy subjects. Data from subjects in the highest and lowest quartiles for motion were used to calculate the interaction between motion and preprocessing. Data were analyzed across four domains of fMRI preprocessing: (1) pipeline, (2) global signal regression (GSR), (3) bandpass filtering, and (4) anatomic atlas. For the FC network calculated from each preprocessing scheme, overall FC using Pearson correlation, as well as leaf fraction and diameter, was calculated for each subject, and statistical comparison was made across schemes using generalized estimating equations.

Results: FC and global network properties were significantly affected by each preprocessing step, and each preprocessing step significantly interacted with subject motion to differentially affect global functional network properties, with GSR having the strongest effect.

Conclusion: Preprocessing choices in fMRI studies influence overall FC and global network properties and can
\end{abstract} have motion-dependent effects.

Keywords: fMRI; functional connectivity; global signal regression; graph theory; preprocessing

\section{Impact Statement}

Different parameter choices across the four domains of functional magnetic resonance imaging (fMRI) preprocessing analyzed in this study (global signal regression, bandpass filtering, anatomic atlas, and pipeline) were associated with differences in functional connectivity (FC) as well as global network properties in healthy subjects. Some fMRI preprocessing parameter choices resulted in motion-dependent effects on FC and global network properties.

\section{Introduction}

A LTHOUGH MEASURING CONNECTIVITY using functional magnetic resonance imaging (fMRI) has become a widely used technique for studying brain activity, there is room for improvement in the reliability, reproducibility, and interpretability of the results of these studies (Noble et al., 2019). One source of variability across functional connectivity (FC) studies using fMRI is the choice of prepro- cessing methodology. Because the blood-oxygen-leveldependent signal is composed of both signal related to neural activity and non-neural sources of variability, various preprocessing techniques have been developed and proposed to identify and reduce the contributions of these non-neural sources as well as to reduce artifacts related to image acquisition and accurately anatomically localize signal.

Commonly used fMRI preprocessing techniques include the following: slice timing correction, signal normalization,

Department of Radiology, Brigham and Women's Hospital, Boston, Massachusetts, USA. 
bandpass filtering, distortion correction, motion correction (including intrasubject registration and regression of motion parameters), regression of other spurious signals (e.g., white matter, cerebrospinal fluid, low-frequency drift, global signal), registration (among standard atlases, anatomic and functional data), and brain tissue segmentation and parcellation using a standard anatomic atlas. For many of these steps, parameters must be chosen by the experimenter (e.g., frequency range for bandpass filtering, choice of anatomic atlas) and often no broad consensus exists about these choices. Similarly, while some of these techniques are broadly used, the use of others remains controversial, such as global signal regression (GSR) (Braun et al., 2012; Jo et al., 2013; Liang et al., 2012; Weissenbacher et al., 2009). Furthermore, both static FC (Gargouri et al., 2018) and dynamic (Vergara et al., 2017) FC, as measured using graph theory, have been shown to be dependent on the order of implementation of preprocessing steps.

Although many software packages and guidelines for fMRI preprocessing exist, most studies involve the use of ad hoc pipelines (Carp, 2012) and the results are presented using only a single set of preprocessing choices. The goal of this study was to study the relationship between different preprocessing schemes and global functional network properties. Although no method currently exists to measure ground truth FC, we compared the robustness of each preprocessing paradigm by measuring changes in betweengroup differences due to subject motion across preprocessing choices. To measure global network FC, we first calculated overall FC, an established metric that has been shown to predictably affect other typically measured graph metrics and between-group network organization if not controlled across subjects (van den Heuvel et al., 2017). We then performed a minimum spanning tree (MST) analysis to extract the network backbone in an unbiased way and calculated two additional global network metrics, leaf fraction and diameter, which quantify network efficiency, integration, and hubness (van Dellen et al., 2018). To our knowledge, no prior study has simul- taneously examined the relationship of each of these preprocessing steps to subject motion and global FC in a large cohort of healthy subjects.

\section{Methods}

\section{Subjects}

Data were obtained from 573 healthy subjects from the publicly available Autism Brain Imaging Data Exchange (ABIDE) database, a set of data released in 2012 from a consortium of 17 international sites (Craddock et al., 2013). Clinical as well as anatomic and resting-state fMRI data were analyzed. Imaging data were incomplete for 65 subjects. Among the remaining 508 subjects (age $=16.4 \pm 7.8$ years, mean \pm SD, $27 \%$ female), motion across each subject's fMRI session was quantified using mean framewise displacement (FD), and the subjects in the highest (mean FD $>0.12 \mathrm{~mm}$, age $=16.2 \pm 8.1$ years) and lowest (mean FD $<0.046 \mathrm{~mm}$, age $=16.7 \pm 7.4$ years) quartiles of motion matched for age and sex were retained for analysis. IRB approval was not necessary for this study as the data are publicly available and anonymized in accordance with the Health Insurance Portability and Accountability Act guidelines. Patient consent was obtained at the time of data collection.

\section{fMRI data}

Scan parameters for anatomic and resting-state functional data are available in detail at the ABIDE I website. Data were obtained across four aspects of preprocessing: (1) pipeline, (2) GSR, (3) bandpass filtering $(0.01-0.1 \mathrm{~Hz})$, and (4) anatomic atlas. The four preprocessing pipelines analyzed were as follows: (1) Connectome Computation System (CCS), (2) Configurable Pipeline for the Analysis of Connectomes (CPAC), (3) Data Processing Assistant for RestingState fMRI (DPARSF), and (4) Neuroimaging Analysis Kit (NIAK), and are summarized in Table 1. Seven anatomic atlases were analyzed: (1) Automated Anatomical Labeling (AAL), (2) Eickhoff-Zilles (EZ), (3) Harvard-Oxford

Table 1. Summary of Parameters of Analysis Pipelines

\begin{tabular}{|c|c|c|c|c|}
\hline Step & $C C S$ & CPAC & $D P A R S F$ & NIAK \\
\hline Drop first volumes & 4 & 0 & 4 & 0 \\
\hline Slice timing correction & Yes & Yes & Yes & No \\
\hline Intensity normalization & 4D global mean & 4D global mean & No & $\begin{array}{l}\text { Nonuniformity correction } \\
\text { using median volume }\end{array}$ \\
\hline Motion regression & 24 parameter & 24 parameter & 24 parameter & $\begin{array}{l}\text { Scrubbing, first principal } \\
\text { component of six motion } \\
\text { parameters and squares }\end{array}$ \\
\hline Tissue regression & Mean WM and CSF & CompCor (5 PCs) & $\begin{array}{l}\text { Mean WM } \\
\text { and CSF }\end{array}$ & Mean WM and CSF \\
\hline Low-frequency drifts & Linear and quadratic & Linear and quadratic & $\begin{array}{l}\text { Linear and } \\
\text { quadratic }\end{array}$ & $\begin{array}{l}\text { Discrete cosine basis with } \\
0.01 \mathrm{~Hz} \text { high-pass cutoff }\end{array}$ \\
\hline $\begin{array}{l}\text { Functional to } \\
\text { anatomical registration }\end{array}$ & $\begin{array}{l}\text { Boundary-based } \\
\text { rigid body }\end{array}$ & $\begin{array}{l}\text { Boundary-based } \\
\text { rigid body }\end{array}$ & Rigid body & Rigid body \\
\hline $\begin{array}{l}\text { Anatomical to } \\
\text { standard registration }\end{array}$ & FLIRT + FNIRT & ANTs & DARTEL & CIVET \\
\hline
\end{tabular}

ANTs, advanced normalization tools; CCS, Connectome Computation System; CPAC, Configurable Pipeline for the Analysis of Connectomes; CSF, cerebrospinal fluid; DARTEL, diffeomorphic anatomical registration through exponential Lie algebra; DPARSF, Data Processing Assistant for Resting-State fMRI; FLIRT, FMRIB's linear image registration tool; fMRI, functional magnetic resonance imaging; FNIRT, FMRIB's nonlinear image registration tool; NIAK, Neuroimaging Analysis Kit; WM, white matter. 
(HO), (4) Talairach and Tournoux (TT), (5) Dosenbach 160 (D160), (6) Craddock 200 (CC200), and (7) Craddock 400 (CC400). Therefore, a total of 112 different preprocessing schemes were analyzed for each subject.

\section{FC networks}

fMRI data were analyzed in MATLAB R2017a (MathWorks, Natick, MA). Overall FC was calculated for each preprocessing scheme as the mean magnitude of the Pearson correlation coefficient across all regions of interest (ROIs). MST were determined for each preprocessing scheme using Prim's algorithm, and the leaf fraction (fraction of nodes with degree 1) and diameter (greatest distance between any pair of nodes) were calculated (van Dellen et al., 2018).

\section{Statistical analysis}

Statistical analysis was performed in R Studio 1.2.5019 (R Studio, Inc., Boston, MA) using the geeglm function of the geepack package. Statistical comparison was made using generalized estimating equations with exchangeable correlation structure and subject identity as the clustering variable. The model formula was as follows:

$$
\begin{gathered}
\text { Connectivity } \sim \text { Age }+ \text { Motion }+ \text { Pipeline }+ \text { GSR }+ \text { Bandpass } \\
+ \text { Atlas }+ \text { Motion } * \text { Pipeline }+ \text { Motion } * \text { GSR } \\
+ \text { Motion } * \text { Bandpass }+ \text { Motion } * \text { Atlas }
\end{gathered}
$$

Where Connectivity represents overall FC, leaf fraction, or diameter. In this model, Age is a continuous variable, while all others are categorical, and the asterisk indicates an interaction term. Information criteria analysis was performed using simplified quasi-information criteria (Pan, 2001) using the QIC function of the geeglm package, which showed that the model with all components had the smallest $\mathrm{QICu}$ value (Supplementary Fig. S1). Correction for multiple comparisons was performed using false discovery rate.

\section{Results}

\section{Effects of preprocessing on overall FC and global properties}

All four domains of preprocessing analyzed in this study were significantly $(p<0.05)$ related to FC and global network properties. GSR (Fig. 1) was associated with 35\% (relative change) lower FC, $4 \%$ lower leaf fraction (LF), and $4 \%$ greater diameter. Bandpass filtering (Fig. 2) was associated with $12 \%$ greater FC and 2\% lower diameter. Pipelines (Fig. 3) were associated with FC (largest to smallest relative values: DPARSF(NS)>CCS(Ref) $>\operatorname{CPAC}(0.93)>\operatorname{NIAK}(0.82)$; $\mathrm{NS}=$ not significantly different from reference), LF (NIAK (1.02) $>$ DPARSF (1.01) $>$ CCS $($ Ref $)>$ CPAC $(0.99)$ ), and diameter $(\mathrm{CPAC}(1.03)>\mathrm{NIAK}(\mathrm{NS})>\mathrm{CCS}(\operatorname{Ref})>\operatorname{DPARSF}(0.98))$. Atlas choice (Fig. 4) was also associated with FC (TT(1.02)>EZ $(\mathrm{NS})>\mathrm{AAL}(\mathrm{Ref})>\mathrm{HO}(0.98)>\mathrm{CC} 400(0.98)>\mathrm{CC} 200(0.89)>$ D160 (0.60), LF (D160(1.17) $>\mathrm{CC} 400(1.06)>\mathrm{HO}(1.04)>$ $\mathrm{TT}(1.03)>\mathrm{CC} 200(1.02)>\mathrm{AAL}(\mathrm{Ref})>\mathrm{EZ}(\mathrm{NS}))$, and diameter $(\mathrm{CC} 400(1.66)>\mathrm{CC} 200(1.28)>\mathrm{EZ}(\mathrm{NS})>\mathrm{AAL}($ Ref $)>\mathrm{D} 160(0.99)$ $>\mathrm{HO}(0.96)>\mathrm{TT}(0.89))$. Finally, greater subject motion was associated with $10 \%$ greater FC, $3 \%$ lower LF, and $6 \%$ greater diameter, and while subject age was not associated with differences in FC, greater age was associated with greater

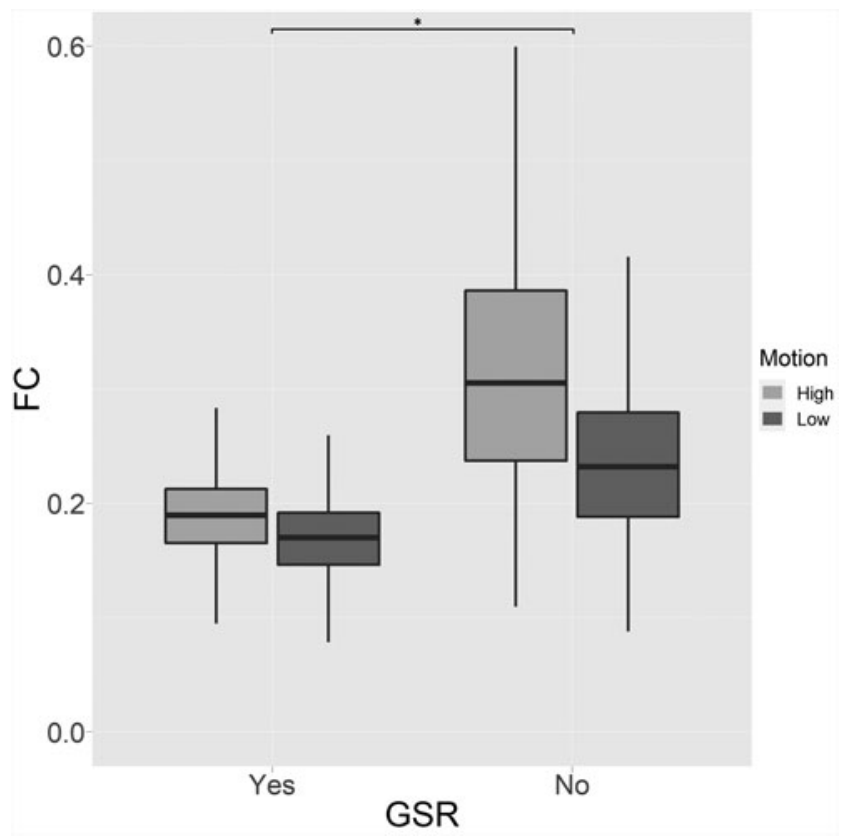

FIG. 1. Global signal regression. Box plots of overall FC values with or without GSR. *Significant $p<0.05$ motiondependent group-wise difference between high and low motion subjects. FC, functional connectivity; GSR, global signal regression.

LF $(0.3 \%$ increase per year of age) and lower diameter $(0.4 \%$ decrease per year of age). Results are summarized in Supplementary Table S1.

\section{Differential effects of preprocessing in high and low motion subjects}

Motion-dependent changes in between-group differences in global network properties and FC across preprocessing

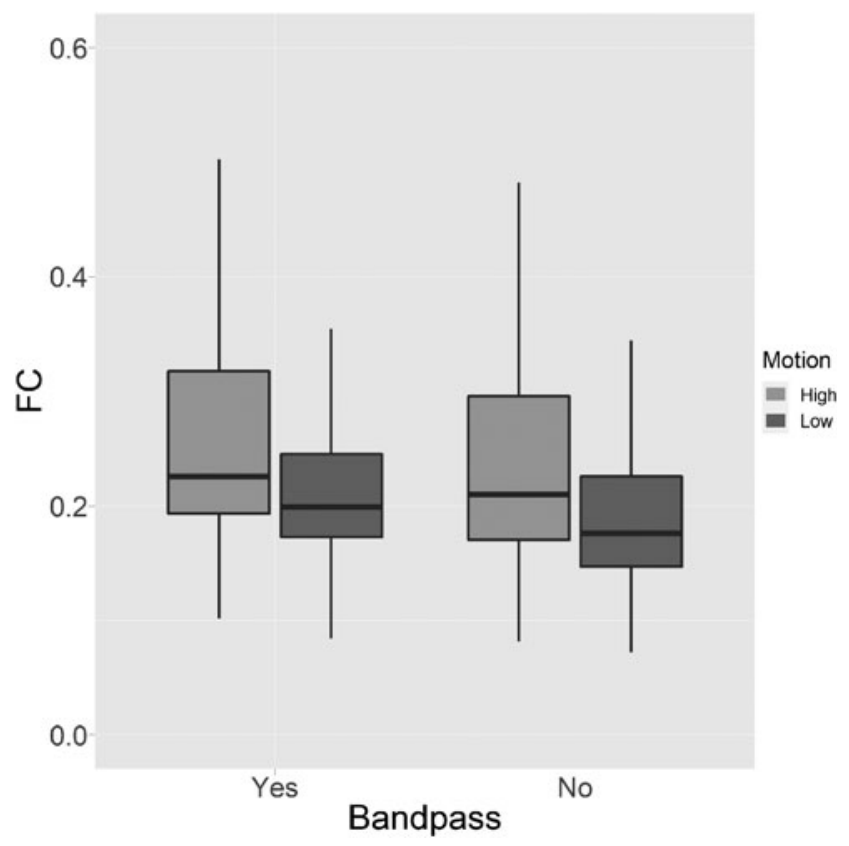

FIG. 2. Bandpass filtering. Box plots of overall FC values with or without bandpass filtering. 


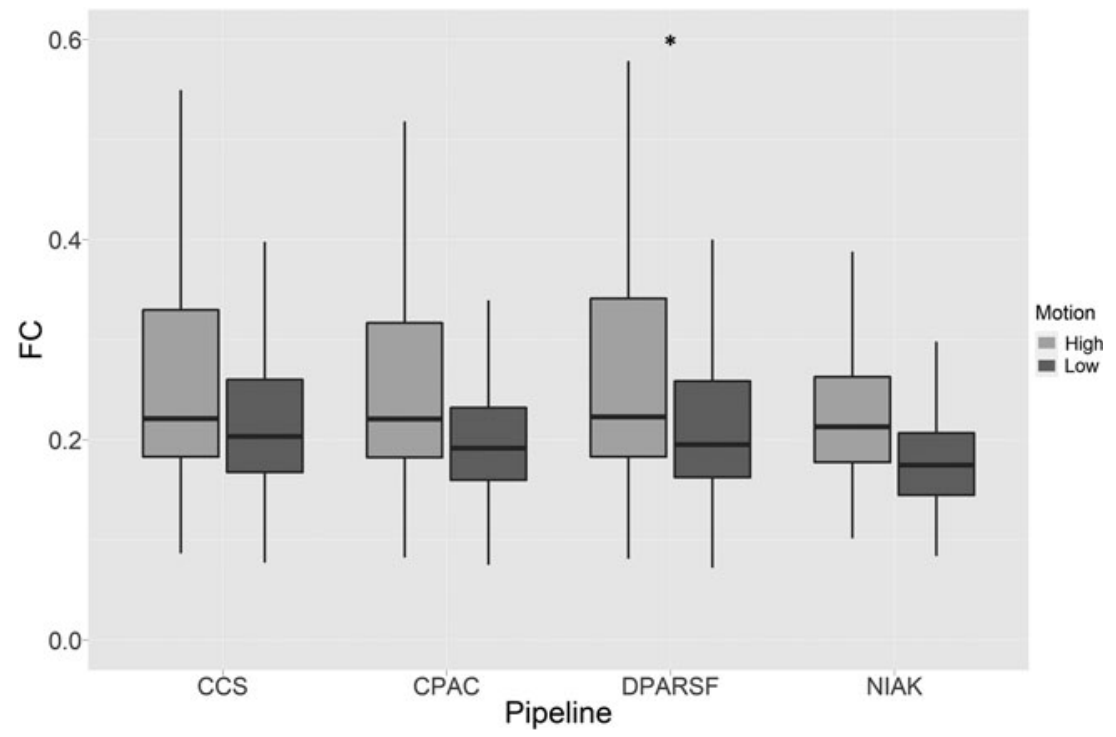

FIG. 3. Pipeline. Box plots of overall FC values across pipelines. *Significant $p<0.05$ motion-dependent group-wise difference between high and low motion subjects. CCS, Connectome Computation System; CPAC, Configurable Pipeline for the Analysis of Connectomes; DPARSF, Data Processing Assistant for RestingState fMRI; fMRI, functional magnetic resonance imaging; NIAK, Neuroimaging Analysis Kit.

choices are summarized in Table 2 . GSR was associated with $73 \%$ lower motion-related difference in FC, 50\% greater motion-related difference in LF, and $110 \%$ greater motionrelated difference in diameter. Only the DPARSF pipeline was associated with a motion-dependent difference in FC, with $3 \%$ greater motion-related difference in FC. The NIAK pipeline was associated with $68 \%$ lower motion-related difference in leaf fraction and $23 \%$ lower motion-related difference in diameter, and the CPAC pipeline was associated with $40 \%$ greater motion-related difference in diameter. Only the D160 atlas was associated with a motion-dependent effect on FC, with 5\% lower motion-related difference in FC. The CC400 atlas was associated with $48 \%$ greater motion-related difference in LF and $135 \%$ greater motion-related difference in diameter, the $\mathrm{HO}$ and TT atlases were associated with $28 \%$ lower motion-related difference in diameter, and the CC200 atlas was associated with $28 \%$ greater motion-related difference in LF. Finally, bandpass filtering did not significantly affect FC with respect to subject motion but was associated with $65 \%$ lower motion-related difference in LF and 36\% lower motion-related difference in diameter.

\section{Discussion}

The results of this analysis demonstrate that global FC network properties vary significantly depending on preprocess- ing methodology, and furthermore that some preprocessing choices differentially affect network properties based on subject motion.

\section{Global signal regression}

In this analysis, GSR had the strongest effect on FC as well as the other global network properties measured and also demonstrated differential effects on high and low motion subjects. The fact that GSR interacts with subject motion is not entirely surprising as the global signal is associated with head motion as well as respiratory and cardiac signals (Liu et al., 2017).

GSR is perhaps the most debated preprocessing step in fMRI analysis, with many studies arguing for and against its routine use. While it improves the specificity of position correlations (Fox et al., 2009), it also introduces spurious anticorrelations (Anderson et al., 2010). In addition, studies have shown both reduced (Guo et al., 2012) and increased (Song et al., 2012) test/retest reliability as well as strengthened ( $\mathrm{Li}$ et al., 2019) and weakened (Gotts et al., 2013) association with behavior with the use of GSR.

The results of this study add to those of prior studies demonstrating that GSR alters graph metrics in restingstate fMRI studies, including both global and local network

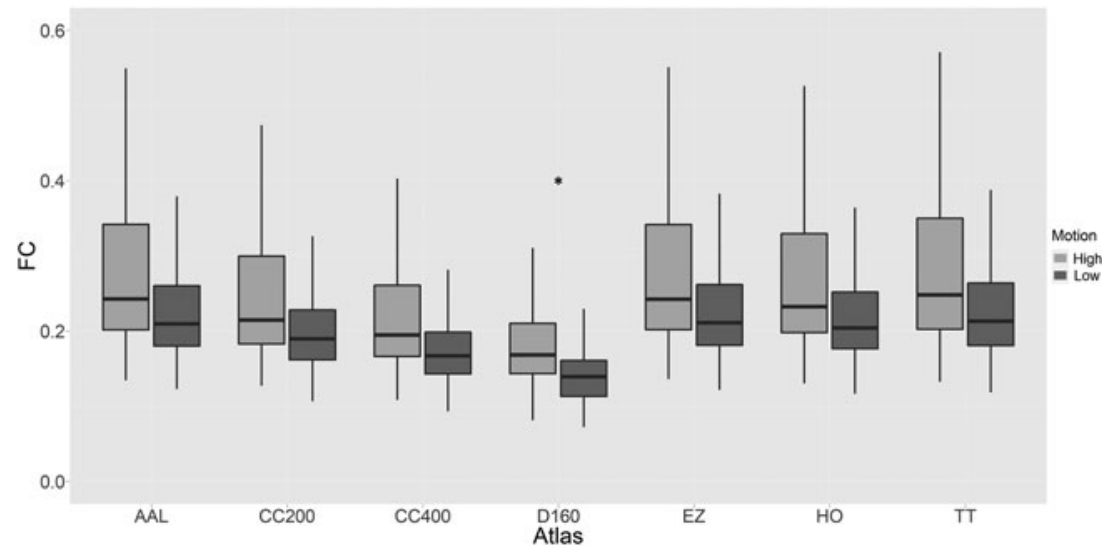

FIG. 4. Atlas. Box plots of overall FC values across atlases. *Significant $p<0.05$ motion-dependent group-wise difference between high and low motion subjects. AAL, Automated Anatomical Labeling; EZ, Eickhoff-Zilles; CC200, Craddock 200; CC400, Craddock 400; D160, Dosenbach 160; HO, HarvardOxford; TT, Talairach and Tournoux. 
TABle 2. Summary of Changes $(P<0.05)$ IN Between-Group Differences of Network Properties Across High and Low Motion SubJects by Preprocessing Methodology

\begin{tabular}{lllll}
\hline Metric & GSR & Bandpass & Atlas & Pipeline \\
\hline FC & $\downarrow$ & - & D160: $\downarrow$ & DPARSF: $\uparrow$ \\
Leaf fraction & $\uparrow$ & $\downarrow$ & CC200: $\uparrow$ & NIAK: $\downarrow$ \\
& & & CC400: $\uparrow$ & \\
Diameter & $\uparrow$ & $\downarrow$ & CC400: $\uparrow$ & CPAC: $\uparrow$ \\
& & & TT: $\downarrow$ & NIAK: $\downarrow$ \\
& & & HO: $\downarrow$ & \\
\hline
\end{tabular}

CC200, Craddock 200; CC400, Craddock 400; D160, Dosenbach 160; FC, functional connectivity; GSR, global signal regression; HO, Harvard-Oxford; TT, Talairach and Tournoux.

properties (Borchardt et al., 2016; Braun et al., 2012; Liang et al., 2012). Liang et al. found that while GSR broadly altered global topological network properties, the spatial distribution of regional hubs was broadly conserved. Borchardt et al. found that GSR had significant effects on raw correlation values, but also global- and local group-level differences between patients with major depressive disorder and healthy patients. Braun et al. observed differences in global network properties, including small-world-ness, network hierarchy, and modularity associated with GSR.

Our results demonstrate that GSR results in lower FC as well as lower variance in FC across subjects, reducing spurious motion-related differences, however, further studies will be necessary to determine if this decrease may come at the cost of masking true group-wise differences under different conditions, and, furthermore, GSR exacerbated motionrelated differences in LF and diameter.

\section{Bandpass filtering}

Of the four domains of preprocessing analyzed in this study, bandpass filtering was found to have the lowest magnitude effect on FC and global network properties, and although motion-dependent difference in FC was not observed, both network diameter and leaf fraction were differentially affected by bandpass filtering with respect to subject motion.

These results are concordant with other studies that have demonstrated that temporal filtering can affect functional network properties, although other studies have compared different frequency ranges rather than the presence or absence of filtering. Liang et al. found that bandpass filtering at different frequency ranges differentially affected global network properties but that regional network properties were relatively preserved. Braun et al. found no differences between broader and narrower band temporal filtering in global network properties, but test/ retest reliability was greater with broader temporal filtering. Borchardt et al. also analyzed three temporal filtering bandwidths and found that while raw correlation values were similar across filtering strategies, temporal filtering differentially affected group-level differences in network properties between patients with major depression and healthy patients.
In summary, bandpass filtering has a modest effect on FC, does not exacerbate or reduce spurious motion-related difference in FC, but does reduce motion-related differences in global network properties.

\section{Anatomic atlas}

Choice of anatomic atlas was significantly associated with FC and global network properties, and for some atlases (D160, CC200, CC400, HO, and TT), motion-dependent differences were observed for either FC or global network properties.

Anatomic atlas choice may be the most heterogeneous preprocessing decision in fMRI analysis methodology, as studies have been performed with ROIs ranging from single voxels to large brain regions. Prior studies have demonstrated that the choice of anatomic atlas, and therefore of network nodal definition, significantly affects both global and local network properties (Korohonen et al., 2017; Wang et al., 2009). Wang et al. found that while smallworld structure was preserved across several atlases, other global and local network properties were affected by atlas choice, including global and local efficiency, characteristic path length, and clustering coefficient. Korhonen et al. found that ROI size is related to consistency of FC and can greatly influence the consistency of metrics derived from these networks.

Unsurprisingly, atlases with more nodes (e.g., CC200, CC400) were associated with larger diameters, but they were also increased motion-dependent differences in global network properties, while atlases with relatively fewer nodes (D160, HO, and TT) were associated with lower motion-dependent differences in FC and network properties.

\section{Pipeline}

We found that different pipelines were associated with differences in FC and global network properties, and that the DPARSF, CPAC, and NIAK pipelines were associated with motion-dependent changes in FC and global properties.

Because these pipelines comprised multiple analysis steps (Table 1), it is difficult to determine the contributions of each substep to these effects on FC and global network properties. Despite this limitation, prior studies have demonstrated that detrending (Borchardt et al., 2016), registration methodology (Conroy et al., 2015; Jiang et al., 2013), and slice-timing correction (Parker and Razlighi, 2019) can influence FC. Borchardt et al. found that while detrending did not strongly affect correlation values, it greatly influenced group differences in network properties between patients with major depression and healthy subjects. Conroy et al. and Jiang et al. found that subtle changes in registration methodology can influence the consistency of FC values. Parker and Razlighi demonstrated that slice-timing correction increased reliability and consistency of FC analysis in both simulated and real fMRI data.

In this analysis, the NIAK pipeline, which lacks slice-timing correction and used a different method for detrending, reduced differential effects on network properties related to patient motion, while the DPARSF and 
CPAC pipelines were associated with small exacerbations in motion-related differences in FC and network properties.

\section{Limitations}

There are several limitations to this study. This study was performed retrospectively and the cohort demographics (predominantly young and male) may limit generalizability of the results of this analysis. This analysis was also limited to global network properties and did not address whether preprocessing choices may have differential effects across brain regions. Furthermore, we limited our analysis to studying FC and graph metrics of connectivity networks but did not explore other common fMRI analysis methodologies such as seed-based analysis, dynamic connectivity, effective connectivity, or independent component analysis.

\section{Conclusions}

Preprocessing choices in fMRI analysis affect overall FC and global functional network properties and may exacerbate motion-dependent differences. Lower motion-related difference was observed when using anatomic atlases with fewer ROIs, when performing bandpass filtering, and using the NIAK pipeline, which would support the use of these preprocessing choices for FC analysis. GSR had the strongest magnitude effect on FC on network properties, reduced motion-related differences in $\mathrm{FC}$, but exacerbated motion-related differences in network properties. Experimenters should interpret between-group differences in FC measured using only a single preprocessing paradigm with caution, and we recommend confirming the robustness of these differences using different preprocessing parameters.

\section{Author Confirmation}

Dr. DeSalvo conceptualized and designed this study, analyzed and interpreted the data, and drafted and approved the final version of publication of this article.

\section{Acknowledgment}

I thank Camden Bay for helpful discussions regarding the statistical methodology for this analysis.

\section{Author Disclosure Statement}

Dr. DeSalvo has no competing interests, personal financial interests, funding, employment, or other competing interests relevant to the current study.

\section{Funding Information}

There is no funding information to declare for this study.

\section{Supplementary Material}

Supplementary Figure S1

Supplementary Table S1

\section{References}

Anderson JS, Druzgal TJ, Lopez-Larson M, et al. 2010. Network anticorrelations, global regression, and phase-shifted soft tissue correction. Hum Brain Mapp 32:919-934.

Borchardt V, Lord AR, Li M, et al. 2016. Preprocessing strategy influences graph-based exploration of altered functional networks in major depression. Hum Brain Mapp 37:1422-1442.

Braun U, Plichta MM, Esslinger C, et al. 2012. Test-retest reliability of resting-state connectivity network characteristics using fMRI and graph theoretical measures. Neuroimage 59:1404-1412.

Carp J. 2012. The secret lives of experiments: methods reporting in the fMRI literature. Neuroimage 63:289-300.

Conroy BR, Singer BD, Haxby JV, et al. 2015. fMRI-based inter-subject cortical alignment using functional connectivity. Adv Neural Inf Process Syst 22:378-386.

Craddock C, Benhajali T, Chu C, et al. The Neuro Bureau Preprocessing Initiative: Open Sharing of Preprocessed Neuroimaging Data and Derivatives. In: Neuroinformatics 2013, Stockholm, Sweden, 2013. http://preprocessedconnectomes-project.org/abide/

Fox MD, Zhang D, Snyder AZ, et al. 2009. The global signal and observed anticorrelated resting state brain networks. J Neurophysiol 101:3270-3283.

Gargouri F, Kallel F, Delphine S, et al. 2018. The influence of preprocessing steps on graph theory measures derived from resting state fMRI. Front Comput Neurosci 12:8.

Gotts SJ, Saad ZS, Jo HJ, et al. 2013. The perils of global signal regression for group comparisons: a case study of autism spectrum disorders. Front Hum Neurosci 7:356.

Guo CC, Kurth F, Zhou J, et al. 2012. One-year test-retest reliability of intrinsic connectivity network fMRI in older adults. Neuroimage 61:1471-1483.

Jiang D, Du Y, Cheng H, et al. 2013. Groupwise spatial normalization of fMRI data based on multi-range functional connectivity patterns. Neuroimage 82:355-372.

Jo HJ, Gotts SJ, Reynolds RC, et al. 2013. Effective preprocessing procedures virtually eliminate distance-dependent motion artifacts in resting state FMRI. J Appl Math 2013: 935154.

Korohonen O, Saarimaki H, Glerean E, et al. 2017. Consistency of regions of interest as nodes of fMRI functional brain networks. Netw Neurosci 1:254-274.

Li J, Kong R, Liegeois R, et al. 2019. Global signal regression strengthens association between resting-state functional connectivity and behavior. Neuroimage 196:126-141.

Liang X, Wang J, Yan C, et al. 2012. Effects of different correlation metrics and preprocessing factors on small-world brain functional networks: a resting-state functional MRI study. PLoS One 7:e32766.

Liu TT, Nalci A, Falahpour M. 2017. The global signal in fMRI: nuisance or information?. Neuroimage 150:213-229.

Noble S, Scheinost D, Constable RT. 2019. A decade of testretest reliability of functional connectivity: a systematic review and meta-analysis. Neuroimage 203:116157.

Pan W. 2001. Akaike's information criterion in generalized estimating equations. Biometrics 57:120-125.

Parker DB, Razlighi QR. 2019. The benefit of slice timing correction in common fMRI preprocessing pipelines. Front NeuroSci 13:821.

Song J, Desphande AS, Meier TB, et al. 2012. Age-related differences in test-retest reliability in resting-state brain functional connectivity. PLoS One 7:e49847. 
van Dellen E, Sommer IE, Bohlken MM, et al. 2018. Minimum spanning tree analysis of the human connectome. Hum Brain Mapp 39:2455-2471.

van den Heuvel MP, de Lange SC, Zalesky A, et al. 2017. Proportional thresholding in resting-state fMRI functional connectivity networks and consequences for patient-control connectome studies: issues and recommendations. Neuroimage 159:437-449.

Vergara VM, Mayer AR, Damaraju E, et al. 2017. The effect of preprocessing in dynamic functional network connectivity used to classify mild traumatic brain injury. Brain Behav 7:e00809.

Wang J, Wang L, Zang Y, et al. 2009. Parcellation-dependent small-world brain functional networks: a resting-state fMRI study. Hum Brain Mapp 30:1511-1523.
Weissenbacher A, Kasess C, Gerstl F, et al. 2009. Correlations and anticorrelations in resting-state functional connectivity MRI: a quantitative comparison of preprocessing strategies. Neuroimage 47:1408-1416.

$$
\begin{array}{r}
\text { Address correspondence to: } \\
\text { Matthew N. DeSalvo } \\
\text { Department of Radiology } \\
\text { Brigham and Women's Hospital } \\
15 \text { Francis Street } \\
\text { Boston, MA 02115-6195 } \\
\text { USA }
\end{array}
$$

E-mail: mdesalvo@bwh.harvard.edu 